\title{
Heart failure - a rare initial manifestation of systemic lupus erythematosus
}

\begin{abstract}
Context: Cardiac involvement is a leading cause of death in systemic lupus erythematosus (SLE). Heart failure (HF) is a potentially life threatening complication of lupus myocarditis which is rarely described as an initial manifestation of SLE. We are presenting a case of HF as initial manifestation of SLE.
\end{abstract}

Case description: 49year old male with no past medical history presented with dyspnea and generalized weakness of 6 weeks duration. Physical exam was remarkable for tachycardia, S3 gallop, bibasilar crackles, and 2+ pitting edema. Echocardiogram showed ejection fraction of $35 \%$ without regional wall motion abnormalities or pericardial effusion. Cardiac catheterization showed normal coronaries. Serological tests indicated positive ANA, Anti-smooth muscle, SCL-70 and SSA antibodies. Patient was given methyl prednisone 1g/day for 3days followed by tapering dose of oral prednisone in addition to intravenous furosemide. He was discharged on prednisone $40 \mathrm{mg}$, hydroxychloroquine $200 \mathrm{mg}$ in addition to B-Blockers and $\mathrm{ACE}$ inhibitors. On follow up evaluation after 4 weeks there was complete resolution of his symptoms with improvement in ejection fraction to $45 \%$.

Conclusion: HF due to myocarditis can be a rare initial presenting manifestation of SLE. Identifying the cause is critical as it dictates therapy. Prompt recognition and a combination of supportive treatment and immunosuppression usually results in a favorable outcome.

Keywords: systemic lupus erythematosus, myocarditis, heart failure, corticosteroids
Volume 5 Issue 3 - 2016

\author{
Hrudya Abraham,' Jose Kuzhively, ${ }^{2}$ Syed \\ $\mathrm{Rizvi}^{3}$ \\ 'Department of Internal Medicine, McNeal Hospital, USA \\ ${ }^{2}$ Department of Endocrinology, Rush University medical Center, \\ USA \\ ${ }^{3}$ Department of Rheumatology, McNeal Hospital, USA
}

\begin{abstract}
Correspondence: Hrudya Abraham, Department of Internal Medicine, McNeal Hospital 3249 S Oak Park Ave, Berwyn-60402, USA, Email drjo999@gmail.com
\end{abstract}

Received: October 31, 2016 | Published: November 28, 2016

\section{Clinical presentation}

49year old male with no past medical history presented with insidious onset dyspnea and generalized weakness of 6 weeks duration. Review of the systems were positive for exertional shortness of breath and leg swelling. Physical exam was remarkable for tachycardia, S3 gallop, bibasilar crackles, and 2+ pitting edema. On admission, he had runs of supraventricular tachycardia that reverted to sinus rhythm with adenosine. Laboratory evaluation was significant for pancytopenia, mild transaminitis (AST>ALT, normal ALP), elevated ESR and CRP. Bone marrow biopsy was normal. Serological tests indicated positive ANA, Anti-smooth muscle, SCL-70 and SSA antibodies. Bone marrow biopsy was normal. Echocardiogram showed ejection fraction of $35 \%$ without regional wall motion abnormalities or pericardial effusion. Cardiac catheterization showed normal coronaries. Since clinical and serological evidence were in accord with SLE, patient was given methyl prednisone $1 \mathrm{~g}$ /day for 3 days followed by tapering dose of oral prednisone in addition intravenous furosemide. Patient was discharged on prednisone $40 \mathrm{mg}$ and hydroxychloroquine $200 \mathrm{mg}$. He was also started on metoprolol and enalapril for his HF. On follow up evaluation in clinic after 4weeks there was complete resolution of his symptoms with improvement in ejection fraction.

\section{Introduction}

Cardiac involvement is a leading cause of death in systemic lupus erythematosus (SLE). Heart failure (HF) is a potentially life threatening complication of lupus myocarditis which is rarely described as an initial manifestation of SLE, especially in an adult. We are reporting a case of SLE with heart failure as initial presentation.
Several auto antibodies, such as anti-phospholipid antibodies, anti-SSA/Ro antibodies and anti-endothelial cells antibodies are also known to mediate cardiac damage. These auto antibodies can either directly affect heart tissue or can trigger mechanisms which can cause cardiac damage. Anti-phospholipid antibodies have been associated with thrombotic events in coronary arteries, heart valve involvement and intra-myocardial vasculopathy in the context of primary and secondary anti-phospholipid syndrome. Antibodies-SSA/Ro and anti$\mathrm{SSB} / \mathrm{La}$ antigens play a major pathogenic role in affecting the heart conduction tissue and have been closely associated with endocardial fibroelastosis.

\section{Discussion}

Cardiac involvement in SLE has been described since the early 20th century. The various manifestations can be from involvement of pericardium, conduction system, myocardium, valves, and coronary arteries. The pathogenesis is thought to be mediated by immune complex formation and complement activation. Focal interstitial plasma cell and lymphocyte infiltrates are seen with fibrinoid degeneration of collagen fibers and small foci of myocardial fibrosis, possibly leading to cardiac dysfunction. Myocarditis is an uncommon manifestation of SLE that can cause heart failure, conduction abnormalities, and arrhythmias. Drug-induced myocarditis should always be excluded in the evaluation of lupus-related myocarditis. Myocardial biopsy is not required in many cases of lupus myocarditis, but can be useful in some patients to confirm the clinical diagnosis, determine the severity of myocardial involvement, and distinguish this disorder from other causes of myocardial disease. However, Endomyocardial biopsy is an invasive procedure and its diagnostic yield is very low at $10-20 \%$. 
Therefore, the diagnosis of myocarditis in SLE depends largely on clinical suspicion, echocardiographic findings and response to steroid therapy. Necropsy studies have shown a prevalence of myocarditis of $40-70 \%$ while symptomatic myocarditis is reported in only $5-10 \%$ of patients, suggesting that subclinical cardiac involvement is a common finding in SLE. Most cases of myocardial dysfunction are indirectly related to contributing factors, including hypertension, coronary artery disease, and medications used to treat SLE. In a case series of 6 patients (1999-2004) with CHF as initial presentation, long term follow-up showed good clinical response with prompt initiation of immunosuppressive therapy. Acute lupus myocarditis merits urgent clinical attention because of the likely progression to arrhythmias, conduction disturbances, dilated cardiomyopathy, and HF. Current treatment strategies are based on clinical experience rather than randomized trials. High-dose glucocorticoid is recommended as initial therapy in addition to treatments for ventricular function (ACE inhibitors, B-blockers and diuretics) is recommended as initial therapy. ${ }^{1-6}$

\section{Conclusion}

HF due to myocarditis can be a rare initial presenting manifestation of SLE. Most reported cases are young patients less than 40year old. Identifying the cause is critical as it dictates therapy. Prompt recognition and a combination of supportive treatment and immunosuppression usually results in a favorable outcome. The present case implicates that lupus myocarditis should be considered in patients with heart failure not responding to conventional therapy and no underlying etiologies identified despite their age.

\section{Conflict of interest}

The authors have nothing to disclose and declares that there is no conflict of interest regarding the publication of this paper. There are no sources of support in the form of grants or industrial support.

\section{References}

1. Van der Laan-Baalbergen NE, Mollema SA, Kritikos H, et al. Heart failure as presenting manifestation of cardiac involvement in systemic lupus erythematosus. Neth J Med. 2009;67(9):295-301

2. Woo SI, Hwang GS, Kang SJ, et al. Lupus myocarditis presenting as acute congestive heart failure: a case report. J Korean Med Sci. 2009;24(1):176-178.

3. Chaudhari D, Madani MA, Balbissi Md KA, et al. Lupus Myocarditis Presenting as Life-threatening Overt Heart Failure:A Case Report with Review of Cardiovascular Manifestations of Systemic Lupus Erythematosus. J La State Med Soc. 2015;167(5):220-222.

4. Inoue Y, Anzawa R, Terao Y, et al. Pericarditis causing congestive heart failure as an initial manifestation of systemic lupus erythematosus. Int $J$ Cardiol. 2007;119(3):e71-e73.

5. Ishimori ML, Agarwal M, Beigel R, et al. Systemic lupus erythematosus cardiomyopathy-a case series demonstrating a reversible form of left ventricular dysfunction. Echocardiography. 2014;31:563-568.

6. Tincani A, Rebaioli CB, Taglietti M, et al. Heart involvement in systemic lupus erythematosus, anti-phospholipid syndrome and neonatal lupus. Rheumatology (Oxford). 2006;45Suppl 4:iv8-13.

\section{Acknowledgements}

None. 